\title{
Post-Optimality Analysis of Energy Consumption Model and Utility Application
}

\author{
Sunday A. Reju' ${ }^{1}$, Gideon Gope ${ }^{2}$ \\ ${ }^{1}$ Department of Mathematics and Statistics, School of Health and Applied Sciences Polytechnic of Namibia \\ (Transforming to Namibia University of Science and Technology), Windhoek, Namibia \\ ${ }^{2}$ Department of Electrical and Computing Engineering, School of Engineering Polytechnic of Namibia \\ (Transforming to Namibia University of Science and Technology), Windhoek, Namibia \\ Email: sreju@polytechnic.edu.na, ggope@polytechnic.edu.na
}

Received 27 October 2014; revised 25 November 2014; accepted 2 December 2014

Copyright (C) 2014 by authors and Scientific Research Publishing Inc.

This work is licensed under the Creative Commons Attribution International License (CC BY).

http://creativecommons.org/licenses/by/4.0/

(c) (;) Open Access

\section{Abstract}

Problems associated with energy distribution, consumption and management are undoubtedly some of the most significant problems that energy utilities face globally. For instance, when development takes place, the demand for electrical power and in particular domestic electrical energy also increases. Thus improvement of energy distribution policies becomes important for utilities and energy decision making agencies. The authors had earlier [1] [2] provided a mixed strategy 2-player game model for a residential energy consumption profile for winter and summer seasons of the year using a dual-occupancy high-rise (11-storey) building located within the Polytechnic of Namibia, Windhoek. The optimum energy values and the corresponding probabilities obtained from the model extend the usual simple statistical analyses of minimum and maximum energy values and their associated percentages. The time-block and the week-day strategies depict critical probabilistic values worth considering for decision purposes, especially, the necessity and justification for a dual tariff regime for the residential and workplace residents of the building as against the existing institutional uniform energy tariff policy. However, this paper presents extended results of post-optimality analyses for the winter and summer seasons, and thus provides the optimal range of energy values over which the energy consumption can change without changing the optimal tariff estimate parameters obtained from the mixed strategy of critical energy game values. The post-optimality analyses also provide extended information on the mixed strategy of non-optimal week-day solutions obtained from the game model, hence validating one of the essential roles of sensitivity analysis, namely, investigation of sub-optimal solutions. From application point of view, the post-optimality model provides a useful tool for Utilities, especially for identifying flexibility range of optimal break-even energy values for consumers, such as in the in- 
formal settlements where metering is rather a challenge to determine varied or non-uniform tariffs.

Keywords

Energy Modeling, Sensitivity Analysis, Post-Optimality, Mixed Strategy Game

\section{Introduction}

Post-optimality Analysis (or Sensitivity Analysis) is concerned with the propagation of uncertainties in mathematical models. It belongs to a broader area of Perturbation Analysis [3] that defines the largest sensitivity region and its main goal is to assess the influence of parameter changes on the state of the system [4]. Post-optimality analysis is necessary in identifying critical or breaking-even values where the optimal strategy changes, and also for investigating sub-optimal solutions. For instance, given the optimal solution of a linear optimization model, a series of post-optimality analyses can provide valuable decision making information to deal with uncertainties.

Specifically, our earlier game model solutions focused on the optimal week-day strategies (or equivalently identified the optimal days of use of energy) and the game value (which was proposed as a uniform tariff estimate parameter). By nature of the game model, there was no information on the energy consumption for the non-optimal week days. The post-optimality analysis model in this paper fills the gap, providing information on the "sub-optimal" solutions which are earlier characterized as "non-optimal" in the game model [1]. Moreover, the dual LPP problem provides the optimal solutions for the time-block decision variables.

We earlier as in [1] solved the following version of the game model represented by Equations (1)-(3):

$$
\text { Maximize } D=\sum_{j=1}^{7} d_{j}
$$

Subject to

$$
\begin{gathered}
\sum_{j=1}^{7} \sum_{i=1}^{48} e_{i j} d_{j} \leq 1 \\
d_{j} \geq 0
\end{gathered}
$$

where

$d_{j}, j=1, \cdots, 7=$ the week day strategies (days of the week, Monday to Sunday).

$e_{i j}=$ the energy consumption values (or the payoff matrix of the game model, represented by the kilowatthour values recorded for each time instant, separated by 30 minutes on each day).

A mixed strategy solution with respective probabilities and value of the game were obtained. However, in this paper, a direct linear programming problem (LPP) approach is employed for the above model to confirm our earlier game model optimal solution and to further derive the associated post-optimality results.

In the following LPP model to be solved, the decision variables $d_{j}$ above have corresponding LPP decision variables as $x 1, x 2, \cdots, x 7$ while the coefficients $e_{i j}$ are as defined above. The relationship between the game model decision variables and the LPP decision variables are defined by Equation (8) below.

\section{Solution of Winter LPP Model}

\subsection{The Model}

Defining the variables $x 1, x 2, x 3, x 4, x 5, x 6$ and $x 7$ as the energy decision variables for the days of the week, namely for Monday, Tuesday..., Sunday, our LPP model is as in Equations (4) and (5):

$$
\text { Maximize } D_{w}=x 1+x 2+x 3+x 4+x 5+x 6+x 7
$$

Subject to 
$27.642678 x 1+28.93933 x 2+29.27172 x 3+28.26516 x 4+28.87478 x 5+28.84201 x 6+30.286526 x 7 \leq 1$ $25.9262393 x 1+25.66183 x 2+26.13624 x 3+27.42176 x 4+26.6133 x 5+28.42685 x 6+28.312744 x 7 \leq 1$ $25.4382561 x 1+25.19901 x 2+25.08198 x 3+25.91629 x 4+25.50332 x 5+25.87914 x 6+25.523228 x 7 \leq 1$ $24.1491054 x 1+23.72513 x 2+24.2098 x 3+24.33872 x 4+25.19087 x 5+23.82306 x 6+23.787366 x 7 \leq 1$ $24.4040223 x 1+23.79068 x 2+24.79247 x 3+23.56741 x 4+23.20033 x 5+23.36639 x 6+23.547017 x 7 \leq 1$ $19.8259667 x 1+22.43399 x 2+23.15372 x 3+22.6366 x 4+22.5929 x 5+23.23311 x 6+23.432911 x 7 \leq 1$ $22.0223721 x 1+22.98421 \times 2+23.10259 x 3+22.98839 x 4+22.78518 x 5+23.1173 x 6+22.949783 x 7 \leq 1$ $22.573477 x 1+22.3307 x 2+22.77863 x 3+22.56887 x 4+23.34454 x 5+23.86676 x 6+22.437522 x 7 \leq 1$ $22.5564828 x 1+22.44392 x 2+23.09346 x 3+22.28045 x 4+23.56086 x 5+23.16756 x 6+23.628677 x 7 \leq 1$ $24.1709557 x 1+22.75976 x 2+23.74102 x 3+22.76333 x 4+21.95925 x 5+22.88569 x 6+24.071417 x 7 \leq 1$ $22.9837729 x 1+22.36646 x 2+22.06632 \times 3+23.44068 x 4+22.42684 x 5+22.74367 x 6+23.231405 x 7 \leq 1$ $23.7728 x 1+22.6366 x 2+22.21053 x 3+23.14134 x 4+21.92429 x 5+22.17338 x 6+22.726427 x 7 \leq 1$ $22.9230771 \times 1+25.40361 \times 2+25.02262 \times 3+23.9913 x 4+23.18941 \times 5+22.66282 x 6+22.175322 x 7 \leq 1$ $25.7562944 x 1+27.08745 x 2+29.76844 x 3+28.51862 x 4+26.87769 x 5+23.59064 x 6+23.105161 x 7 \leq 1$ $32.614771 \times 1+34.9753 x 2+36.61842 x 3+33.93305 x 4+34.17777 x 5+25.03355 x 6+23.607711 x 7 \leq 1$ $38.065133 x 1+45.54415 x 2+43.31764 x 3+42.36934 x 4+41.25718 x 5+28.10129 x 6+27.42175 x 7 \leq 1$ $45.229496 x 1+51.04597 x 2+52.69783 x 3+49.28924 x 4+48.61626 x 5+31.19307 x 6+41.94737 x 7 \leq 1$ $45.363028 x 1+47.52158 x 2+46.50336 x 3+47.25937 x 4+47.40795 x 5+37.14499 x 6+38.01901 x 7 \leq 1$ $41.762636 \times 1+44.0933 \times 2+43.43125 \times 3+44.13264 \times 4+42.70802 \times 5+39.21589 x 6+39.30815 \times 7 \leq 1$ $40.985743 x 1+40.35914 x 2+40.10131 \times 3+38.48182 x 4+37.75243 \times 5+39.86168 x 6+41.79906 x 7 \leq 1$ $40.160296 x 1+37.44654 \times 2+36.44799 x 3+36.48354 x 4+38.62644 x 5+40.30111 x 6+41.04887 x 7 \leq 1$ $36.899789 x 1+36.74972 x 2+36.67087 x 3+34.82095 x 4+38.37953 x 5+39.13578 x 6+40.9906 x 7 \leq 1$ $35.0935278 x 1+35.64729 x 2+35.53904 x 3+34.39191 x 4+36.21857 x 5+37.82477 x 6+37.2761 x 7 \leq 1$ $34.930871 x 1+34.3641 x 2+35.61551 \times 3+35.36919 x 4+34.44435 x 5+37.31737 x 6+37.77622 x 7 \leq 1$ $33.3018256 x 1+34.49519 x 2+32.94325 x 3+35.66715 x 4+33.20983 x 5+37.91703 x 6+35.236767 x 7 \leq 1$ $33.430505 x 1+33.55166 x 2+32.2659 x 3+33.60928 x 4+33.74951 x 5+37.22755 x 6+37.873333 x 7 \leq 1$ $33.024095 x 1+34.05819 x 2+34.68252 x 3+32.31417 x 4+32.23969 x 5+37.74708 x 6+36.465216 x 7 \leq 1$ $33.568155 x 1+35.23677 x 2+35.12825 x 3+33.48413 x 4+33.84129 x 5+34.98913 x 6+34.24623 x 7 \leq 1$ $34.673775 x 1+34.2171 \times 2+34.1734 x 3+33.62317 x 4+34.44435 x 5+34.90174 x 6+34.1297 x 7 \leq 1$ $32.746595 x 1+31.30012 x 2+33.767 x 3+34.85671 x 4+35.44727 x 5+35.06682 x 6+34.06172 x 7 \leq 1$ $31.01389 x 1+31.62788 x 2+32.66358 x 3+30.74096 x 4+31.97748 x 5+32.74344 x 6+35.68106 x 7 \leq 1$ $30.327805 x 1+29.8562 x 2+29.70871 x 3+29.10008 x 4+30.01972 x 5+31.41059 x 6+33.58102 x 7 \leq 1$ $28.66502 x 1+30.08563 x 2+29.79612 x 3+28.48843 x 4+29.77937 x 5+31.78689 x 6+30.194267 x 7 \leq 1$ $30.59219 x 1+29.56305 x 2+28.78738 x 3+28.68905 x 4+29.15665 x 5+31.21394 x 6+32.78471 x 7 \leq 1$ $29.195975 x 1+30.99422 x 2+30.37587 x 3+28.05739 x 4+29.04521 \times 5+30.83521 x 6+30.429767 x 7 \leq 1$ $31.608215 x 1+31.32381 \times 2+29.39262 \times 3+30.20464 x 4+31.07071 \times 5+31.97141 \times 6+32.493379 x 7 \leq 1$ $33.41968 x 1+32.87151 x 2+34.00517 x 3+32.4552 x 4+31.61696 x 5+33.14646 x 6+33.96704 x 7 \leq 1$ $36.316885 x 1+36.73714 x 2+38.2681 x 3+35.5401 \times 4+34.25426 x 5+35.5718 x 6+36.33655 x 7 \leq 1$ $39.943995 x 1+41.30196 x 2+43.45747 x 3+39.03205 x 4+37.44435 x 5+40.12631 x 6+38.48514 x 7 \leq 1$ $39.489515 x 1+43.45419 x 2+41.99133 x 3+40.99656 x 4+41.2965 x 5+38.38608 x 6+40.30839 x 7 \leq 1$ $41.99352 \times 1+43.52339 \times 2+41.36861 \times 3+41.00251 \times 4+40.57109 x 5+40.02678 x 6+39.09693 \times 7 \leq 1$ $39.31252 x 1+40.20218 x 2+40.76992 x 3+37.92875 x 4+38.98478 x 5+39.36642 x 6+38.77889 x 7 \leq 1$ $36.66212 x 1+40.96328 x 2+38.13918 x 3+37.51646 x 4+37.15374 x 5+36.78326 x 6+40.3351 \times 7 \leq 1$ $39.382445 x 1+39.14245 x 2+36.62498 x 3+36.95232 x 4+37.25426 x 5+35.2052 x 6+34.62254 x 7 \leq 1$ $36.51354 x 1+37.66576 \times 2+35.01901 \times 3+36.11011 \times 4+36.84129 x 5+35.58152 x 6+33.61501 \times 7 \leq 1$ $34.582005 x 1+34.40829 x 2+33.92213 x 3+33.63311 x 4+35.07581 x 5+32.3987 x 6+32.2506 x 7 \leq 1$ $32.59802 x 1+34.05687 x 2+33.72548 x 3+31.59708 x 4+33.1836 x 5+31.78933 x 6+31.092551 \times 7 \leq 1$ $29.34018 x 1+32.81142 x 2+30.39554 x 3+30.44102 x 4+31.23021 \times 5+32.83812 x 6+29.762126 x 7 \leq 1$ 
The coefficients in the above model are the energy readings taken from the dual-occupancy high-rise building (stated in the Abstract).

The optimal solution for the above model is as follows

$$
\begin{aligned}
& x 1=0 \\
& x 2=0 \\
& x 3=0 \\
& x 4=0.00233375565998705 \\
& x 5=0.00196693868495645 \\
& x 6=0.0155408776995797 \\
& x 7=0.00517836109162509
\end{aligned}
$$

And

$$
D_{w}=0.0250199331361483
$$

Theoretically, given the expected value of the game defined by Equation (6) in our previous game model [1]

$$
T E D=\sum_{j=1}^{7} \sum_{i=1}^{48} e_{i j} t_{i} X_{j}=p
$$

where $T$ is the time strategy vector, $E=e_{i j}$, the energy payoff matrix and $X_{j}$ the Day strategy vector, once we solve the associated linear programming problem, the value of the game is obtained by the formula in Equation (7) below.

$$
p=\frac{1}{D_{w}}
$$

Thus, we have

$$
p=\frac{1}{0.0250199}=39.96818532
$$

Moreover, the column player's (week day) optimal mixed strategy is obtained by the formula in Equation (8):

$$
d_{j}=\frac{X_{j}}{D_{w}}
$$

where $X_{j}=x j, \quad j=1,2,3, \cdots, 7$

The comparative results are provided in Table 1.

It is observed that the above probabilities coincide with those earlier obtained from our game model [1] in Table 2.

\subsection{Winter Week-Day Sensitivity Analysis Results}

Figure 1 summarises the procedure for the post-optimality analysis and Table 3 is the result of running a PostOptimality test based on the Simplex method, using the LPSolve package.

The above gives on dividing by $D_{w}=0.025019933$, the optimal range of energy values as follows (and using zero (0) in place of $-\infty$ for non-negative energy values):

$$
\begin{gathered}
0 \leq X_{1} \leq 41.3948284 \\
0 \leq X_{2} \leq 41.72583365 \\
0 \leq X_{3} \leq 40.29555199
\end{gathered}
$$

$39.75519492 \leq X_{4} \leq 40.56906598$

$39.61080734 \leq X_{5} \leq 40.28072835$

$39.42965009 \leq X_{6} \leq 40.19669252$ 
Table 1. Winter LPP and game model comparison.

\begin{tabular}{ccc}
\hline & LPP Model & Game Model (2012) \\
\hline Optimal Objective & $D_{w}=0.025019933$ & $p=\frac{1}{D_{w}}=39.96813239$ \\
\hline Variables & $X_{j}$ & $\frac{X_{j}}{D_{w}}=d_{j}$ \\
\hline$X_{1}$ & 0 & Probabilities \\
$X_{2}$ & 0 & 0 \\
$X_{3}$ & 0 & 0 \\
$X_{4}$ & 0.002333756 & 0 \\
$X_{5}$ & 0.001966939 & 0.093275856 \\
$X_{6}$ & 0.015540878 & 0.078614866 \\
$X_{7}$ & 0.005178361 & 0.621139861 \\
\hline
\end{tabular}

\section{$39.50194189 \leq X_{7} \leq 40.86035541$}

The $5^{\text {th }}$ degree polynomial regression plots for the optimal range of values (with the week day mean plot for the winter season) are as in Figure 2.

It is observed that the four-day optimal energy consumption days for the season (Thursday-Sunday) as obtained in our game model show fairly stable consumption profile from the post-optimality results (i.e. the values showing very close proximity).

Superposed plots with the mixed-strategy game model tariff estimate value are shown in Figure 3.

\subsection{Winter Time Block Sensitivity Analysis Results}

The following time-block optimal range of values in Table 4 which coincide with the same optimal time-blocks in the game model is obtained from the Dual problem (using the LPSolve Software).

Using Equation (6), we obtain the corresponding post-optimality energy ranges by computing the values using Equation (9).

$$
T_{i}=\frac{t_{i}}{T_{w}}
$$

where $T_{i}$ is the Time-Block optimal mixed-strategy and $T_{w}=D_{w}$

Thus the ranges of energy values are as follows:

$$
\begin{aligned}
& 39.73552664 \leq t_{19} \leq 40.25188749 \\
& 39.59374664 \leq t_{20} \leq 40.16628286 \\
& 39.80201684 \leq t_{21} \leq 40.21069659 \\
& 39.58727316 \leq t_{41} \leq 40.15870528
\end{aligned}
$$

where $t_{19}, t_{20}, t_{21}$, and $t_{41}$ are the time instants indicated in Table 4.

\section{Solution of Summer LPP Model}

\subsection{The LPP Model}

Following similar procedure as in the winter model, we have the summer linear programming model given by Equations (10) and (11):

$$
\text { Maximize } D_{s}=x 1+x 2+x 3+x 4+x 5+x 6+x 7
$$

Subject to 
$23.8688 x 1+23.8274 x 2+23.2569 x 3+22.9328 x 4+22.0727 x 5+23.0904 x 6+24.1528 x 7 \leq 1$ $23.1384 x 1+22.3498 x 2+22.3793 x 3+23.0153 x 4+22.6224 x 5+22.8303 x 6+22.8204 x 7 \leq 1$ $22.7459 x 1+22.1436 x 2+23.2266 x 3+22.2651 x 4+21.6950 x 5+23.1991 x 6+22.4068 x 7 \leq 1$ $22.0477 x 1+22.2351 x 2+21.6643 x 3+21.6922 x 4+21.5899 x 5+22.6435 x 6+23.2614 x 7 \leq 1$ $20.5729 x 1+21.7858 x 2+21.8658 x 3+21.8245 x 4+21.0303 x 5+21.5025 x 6+23.1889 x 7 \leq 1$ $22.6055 x 1+21.8650 x 2+21.5271 \times 3+21.4470 x 4+22.1830 x 5+21.5609 x 6+22.4373 x 7 \leq 1$ $21.0313 x 1+21.8514 x 2+22.8272 x 3+22.0891 x 4+20.8742 x 5+20.7814 x 6+22.5856 x 7 \leq 1$ $22.0382 x 1+21.5755 x 2+21.7675 x 3+21.2977 x 4+21.0296 x 5+20.5841 x 6+21.6074 x 7 \leq 1$ $21.5296 x 1+21.9442 x 2+21.1411 x 3+22.2190 x 4+20.8120 x 5+22.0394 x 6+21.0020 x 7 \leq 1$ $22.0616 x 1+21.7093 x 2+23.2338 x 3+22.7058 x 4+22.3151 x 5+20.2957 x 6+21.9485 x 7 \leq 1$ $22.1518 x 1+23.4191 x 2+24.1904 x 3+24.0471 x 4+24.5878 x 5+21.8365 x 6+21.8885 x 7 \leq 1$ $26.2768 x 1+27.2196 x 2+28.4681 x 3+29.3033 x 4+28.6658 x 5+22.3676 x 6+21.7619 x 7 \leq 1$ $30.7296 x 1+32.9430 x 2+32.7119 x 3+34.4842 x 4+34.0827 x 5+23.0365 x 6+22.4974 x 7 \leq 1$ $36.4062 \times 1+39.1675 \times 2+42.2251 \times 3+40.9433 x 4+38.8082 x 5+25.6101 \times 6+25.8903 x 7 \leq 1$ $44.4838 x 1+43.8093 \times 2+40.0826 x 3+44.5303 x 4+42.8277 x 5+29.4292 x 6+27.5326 x 7 \leq 1$ $37.2820 x 1+39.6564 x 2+40.7454 x 3+40.1300 x 4+40.3427 x 5+34.2710 x 6+31.8429 x 7 \leq 1$ $33.5061 x 1+34.9832 x 2+37.5067 x 3+35.4358 x 4+35.9067 x 5+34.7583 x 6+25.3363 x 7 \leq 1$ $32.1347 x 1+33.3199 x 2+34.9466 x 3+34.5898 x 4+34.6139 x 5+34.6564 x 6+37.4958 x 7 \leq 1$ $32.2935 x 1+32.7914 x 2+31.6716 x 3+31.6230 x 4+32.0852 x 5+34.9456 x 6+36.6587 x 7 \leq 1$ $30.3029 x 1+33.4100 x 2+30.2847 x 3+30.8952 x 4+31.6720 x 5+38.4210 x 6+33.9243 x 7 \leq 1$ $32.0694 \times 1+31.0215 \times 2+32.0066 x 3+31.5910 \times 4+31.2710 x 5+35.1093 \times 6+34.3863 \times 7 \leq 1$ $32.6619 x 1+31.7016 x 2+30.6373 x 3+30.5602 x 4+29.2036 x 5+35.7201 x 6+33.1212 x 7 \leq 1$ $33.2202 x 1+33.2513 \times 2+28.8423 x 3+31.4552 x 4+29.9035 x 5+35.9908 x 6+34.0931 \times 7 \leq 1$ $33.3745 \times 1+32.3060 \times 2+31.0331 \times 3+31.9017 x 4+29.5993 \times 5+34.6630 \times 6+34.1961 \times 7 \leq 1$ $33.0741 \times 1+30.5026 x 2+30.9420 x 3+32.1767 x 4+28.7542 x 5+35.3256 x 6+33.3463 x 7 \leq 1$ $32.9157 x 1+30.5962 x 2+29.8386 x 3+31.1844 x 4+29.9234 x 5+32.8237 x 6+33.7195 x 7 \leq 1$ $34.7797 x 1+32.7478 x 2+32.7313 x 3+31.8587 x 4+31.0841 x 5+32.7131 x 6+33.2156 x 7 \leq 1$ $32.2069 x 1+31.5432 \times 2+32.0661 \times 3+30.8912 x 4+29.9343 \times 5+32.1901 \times 6+32.2649 \times 7 \leq 1$ $30.8112 x 1+29.9695 x 2+29.8010 x 3+29.2615 x 4+29.5747 x 5+32.1705 x 6+30.4433 x 7 \leq 1$ $30.0997 x 1+27.1236 x 2+27.6900 x 3+28.6936 x 4+27.5468 x 5+31.1264 x 6+29.9617 x 7 \leq 1$ $29.8034 x 1+28.7377 x 2+26.6000 x 3+26.7959 x 4+25.6304 x 5+31.1609 x 6+32.5136 x 7 \leq 1$ $31.6647 x 1+27.1587 x 2+28.6963 x 3+26.2556 x 4+28.4445 x 5+31.6943 x 6+29.3786 x 7 \leq 1$ $28.1087 x 1+26.4226 x 2+28.5040 x 3+26.6707 x 4+36.9958 x 5+30.4599 x 6+30.3961 x 7 \leq 1$ $27.9339 x 1+28.3682 x 2+28.7789 x 3+28.4718 x 4+27.7238 x 5+30.8574 x 6+29.9319 x 7 \leq 1$ $29.8498 x 1+29.7899 \times 2+28.5834 x 3+28.9063 x 4+30.6831 \times 5+30.0002 x 6+30.7471 x 7 \leq 1$ $32.5346 x 1+32.0640 x 2+30.7927 x 3+30.6895 x 4+32.9890 x 5+31.9891 \times 6+31.4353 x 7 \leq 1$ $35.2085 x 1+33.1100 x 2+33.1756 x 3+32.4497 x 4+34.3692 x 5+31.4413 x 6+31.3976 x 7 \leq 1$ $34.8207 x 1+34.5622 x 2+34.7002 x 3+35.5451 x 4+36.0338 x 5+32.5638 x 6+34.7076 x 7 \leq 1$ $36.4513 x 1+36.8359 \times 2+37.4703 x 3+36.2188 x 4+34.5814 x 5+34.2888 x 6+34.7784 x 7 \leq 1$ $37.2543 \times 1+34.9004 \times 2+37.8818 x 3+37.3004 x 4+34.8331 \times 5+33.1371 x 6+36.5130 \times 7 \leq 1$ $37.7609 x 1+34.7049 \times 2+37.7119 x 3+37.9353 x 4+36.0665 x 5+34.7353 x 6+36.3382 x 7 \leq 1$ $35.4530 \times 1+34.1571 \times 2+37.7944 \times 3+37.7750 \times 4+34.0789 \times 5+33.1041 \times 6+36.9849 \times 7 \leq 1$ $36.0047 x 1+33.1094 x 2+34.2426 x 3+34.9891 x 4+33.0504 x 5+32.7393 x 6+34.7846 x 7 \leq 1$ $32.4036 x 1+33.6719 \times 2+33.0700 x 3+32.6658 x 4+33.6535 x 5+31.9995 x 6+32.9181 \times 7 \leq 1$ $32.3503 x 1+28.3440 x 2+29.0714 x 3+30.3921 x 4+29.6728 x 5+30.5725 x 6+31.6519 x 7 \leq 1$ $29.5876 x 1+26.8952 x 2+29.0896 x 3+29.2341 x 4+27.5456 x 5+29.2795 x 6+29.1900 x 7 \leq 1$ $27.0080 x 1+25.5740 x 2+25.7405 x 3+27.5395 x 4+25.8128 x 5+28.9487 x 6+27.0588 x 7 \leq 1$ $24.9841 x 1+25.0066 x 2+25.4480 x 3+25.2465 x 4+25.5130 x 5+25.5799 x 6+25.7625 x 7 \leq 1$ 
Table 2. Winter energy consumption concentration matrix.

\begin{tabular}{cccccc}
\hline & Days & Thu & Fri & Sat & Sun \\
\hline Time & Probabilities & 0.0933 & 0.0786 & 0.6211 & 0.2070 \\
\hline 09.00 & 0.1208 & 44.1326 & 42.7080 & 39.21589 & 39.3082 \\
09.30 & 0.1460 & 38.4818 & 37.7524 & 39.86168 & 41.7991 \\
10.00 & 0.2311 & 36.4835 & 38.6264 & 40.30111 & 41.0489 \\
20.00 & 0.5021 & 41.0025 & 40.5711 & 40.02678 & 39.0969 \\
Game Value & & & 39.9681 & & \\
\hline
\end{tabular}

Table 3. Results of winter week-day sensitivity analysis.

\begin{tabular}{ccc}
\hline & From & Till \\
\hline Objective & 0.0250199331361483 & 0.0250199331361483 \\
\hline Variables & $-\infty$ & 1.03569583315223 \\
\hline$X_{1}$ & $-\infty$ & 1.04397756219379 \\
$X_{2}$ & $-\infty$ & 1.00819201095926 \\
$X_{3}$ & 0.994672313337416 & 1.01503531275992 \\
$X_{4}$ & 0.991059745754735 & 1.00782112455324 \\
$X_{5}$ & 0.986527203511101 & 1.00571855368084 \\
$X_{6}$ & 0.98833593943488 & 1.02232335482563 \\
$X_{7}$ & & \\
\hline
\end{tabular}
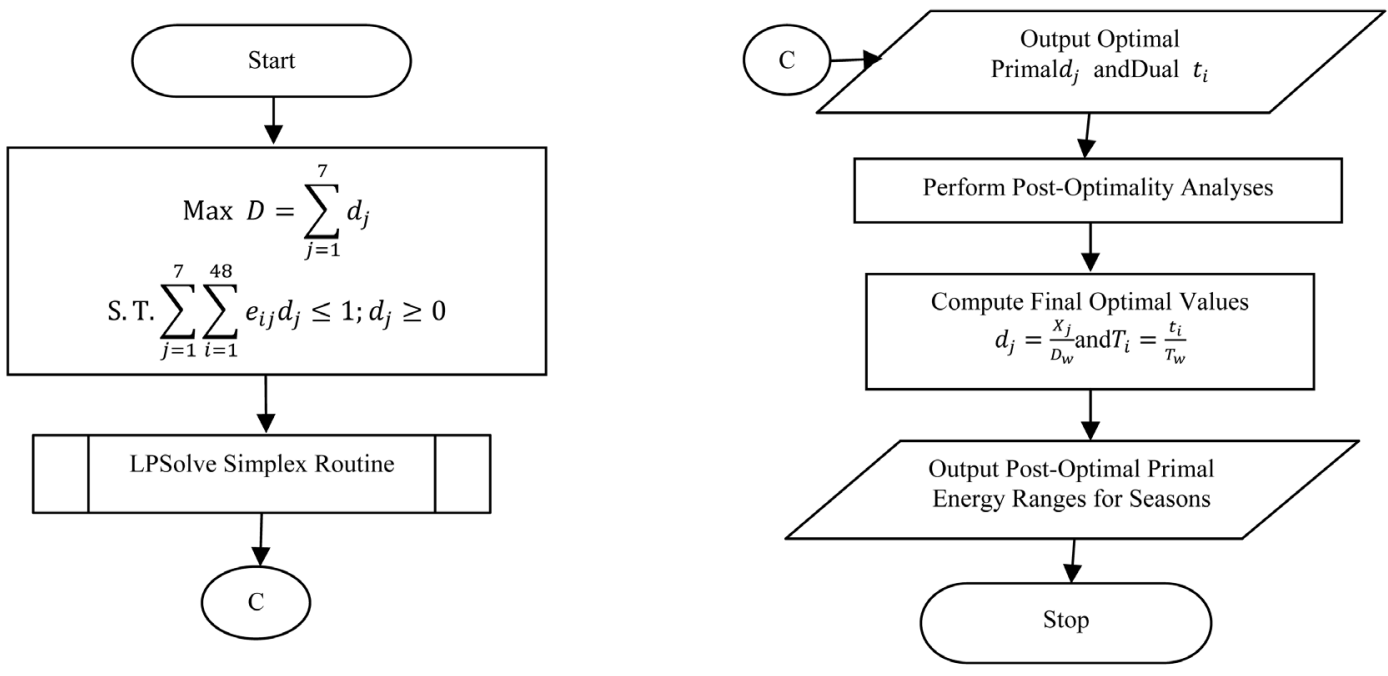

Figure 1. Post-Optimality algorithm for winter model.

The optimal solution is as follows:

$$
\begin{aligned}
& x 1=0 \\
& x 2=0.00906091473154742 \\
& x 3=0 \\
& x 4=0 \\
& x 5=0.000330919431198716 \\
& x 6=0.00966675499545442 \\
& x 7=0.00929680391087571
\end{aligned}
$$




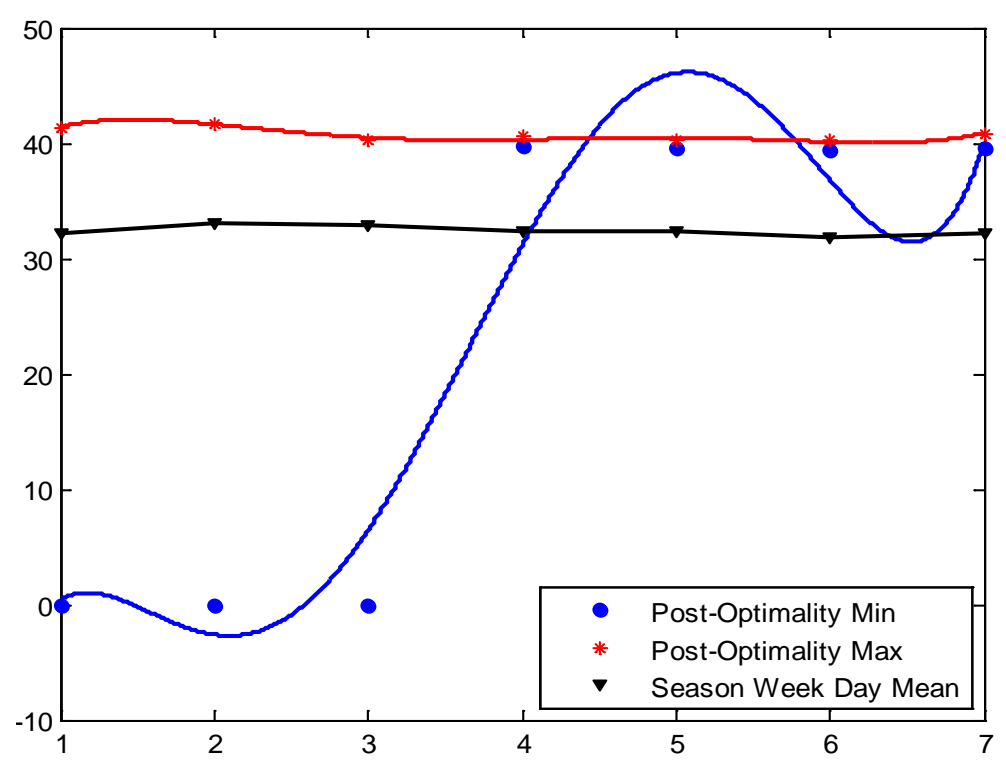

Figure 2. Relationships between the seasonal mean and post-optimality energy values.

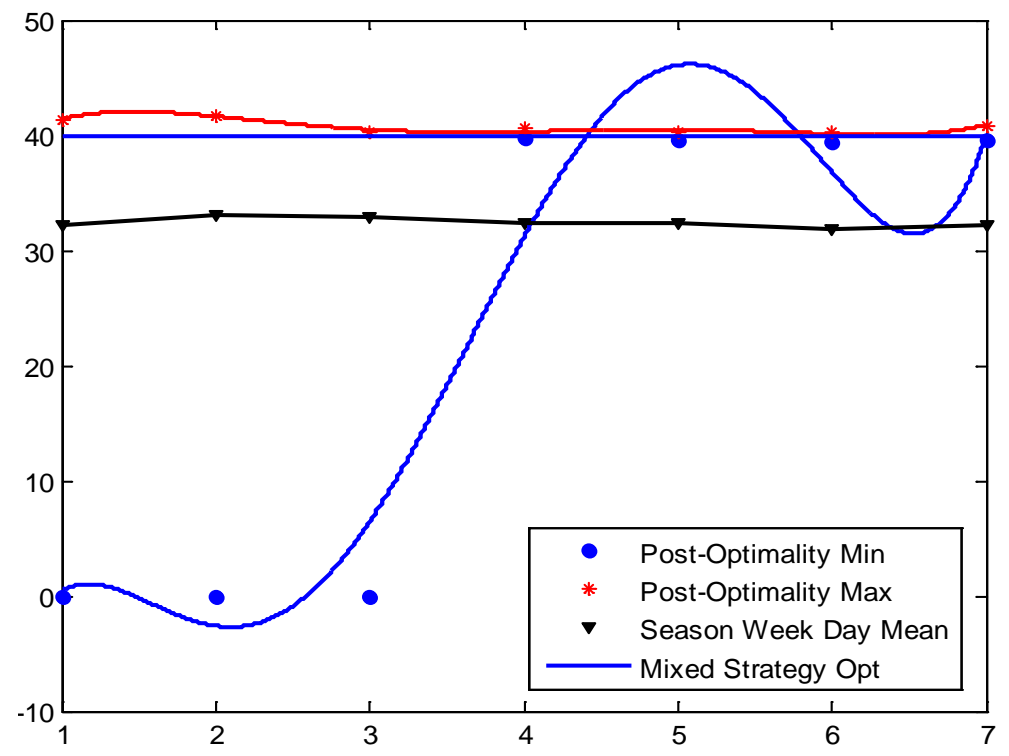

Figure 3. Relationships between the seasonal mean, post-optimality energy values and the tariff estimator.

Table 4. Results of winter dual problem sensitivity analysis.

\begin{tabular}{ccc}
\hline & From & Till \\
\hline Objective & 0.0250199331361483 & 0.0250199331361483 \\
\hline Variables & & \\
\hline$t_{19}=09 \mathrm{H} 00$ & 0.99418022 & 1.007099534 \\
$t_{20}=09 \mathrm{H} 30$ & 0.990632894 & 1.004957712 \\
$t_{21}=10 \mathrm{H} 00$ & 0.9958438 & 1.00606894 \\
$t_{41}=20 \mathrm{H} 00$ & 0.990470928 & 1.004768121 \\
\hline
\end{tabular}


and

$$
D_{s}=0.0283553930690763
$$

For the summer season, the value of the game is obtained using Equation (12) below:

$$
p=\frac{1}{D_{s}}
$$

Thus, we have

$$
p=\frac{1}{0.0283553930}=35.26665986
$$

The comparative table of results for the summer season is as follows in Table 5:

It is also observed that the above probabilities coincide with those earlier obtained from our game model [1] for the season as seen in Table 6.

\subsection{Summer Week-Day Sensitivity Analysis Results}

Running the LPSolve Sensitivity Analysis routine for the problem, we have the results as presented in Table 7.

On dividing by $D_{s}=0.028355393$, the above gives the optimal range of energy values as follows (using zero (0) in place of $-\infty$ for non-negative energy values):

Table 5. Summer LPP and game model comparison.

\begin{tabular}{|c|c|c|c|c|c|}
\hline & Days & Tue & Fri & Sat & Sun \\
\hline Time & Probabilities & 0.3195 & 0.0117 & 0.3409 & 0.3279 \\
\hline 7.30 & 0.0695 & 39.65638 & 40.34272 & 34.27103 & 31.8429 \\
\hline 9.30 & 0.1784 & 33.41002 & 31.67203 & 38.42097 & 33.92427 \\
\hline 19.00 & 0.2106 & 36.835885 & 34.58144 & 34.28885 & 34.77837 \\
\hline 20.00 & 0.5415 & 34.704865 & 36.06655 & 34.73525 & 36.33819 \\
\hline Value & & & 35.2666 & & \\
\hline
\end{tabular}

\begin{tabular}{ccc}
\hline & LPP Model & Game Model \\
\hline Optimal Objective & $D_{s}=0.028355393$ & $p=\frac{1}{D_{s}}=35.26665986$ \\
\hline Variables & $X_{j}$ & $\frac{X_{j}}{D_{s}}$ \\
\hline$X_{1}$ & 0 & Probabilities \\
$X_{2}$ & 0.009060915 & 0 \\
$X_{3}$ & 0 & 0.319548198 \\
$X_{4}$ & 0 & 0 \\
$X_{5}$ & 0.000330919 & 0 \\
$X_{6}$ & 0.009666755 & 0.011670423 \\
$X_{7}$ & 0.009296804 & 0.34091416 \\
\hline
\end{tabular}

Table 6. Summer energy consumption concentration matrix. 
Table 7. Results of summer week-day sensitivity analysis.

\begin{tabular}{ccc}
\hline Variables & From & Till \\
\hline Objective & 0.0283553930690763 & 0.0283553930690763 \\
\hline$X_{1}$ & $-\infty$ & 1.02422635412771 \\
$X_{2}$ & 0.981537627468657 & 1.06062733133012 \\
$X_{3}$ & $-\infty$ & 1.03629009420123 \\
$X_{4}$ & $-\infty$ & 1.03412262609405 \\
$X_{5}$ & 0.982292128971773 & 1.02150606857908 \\
$X_{6}$ & 0.969765665592283 & 1.03416157676571 \\
$X_{7}$ & 0.974735091792164 & 1.0145564767584 \\
\hline
\end{tabular}

$$
\begin{aligned}
& 0 \leq X_{1} \leq 36.12104245 \\
& 34.61555364 \leq X_{2} \leq 37.40478333 \\
& 0 \leq X_{3} \leq 36.54649026 \\
& 0 \leq X_{4} \leq 36.4700509 \\
& 34.64216239 \leq X_{5} \leq 36.02510706 \\
& 34.20039587 \leq X_{6} \leq 36.47142456 \\
& 34.37565093 \leq X_{7} \leq 35.78001817
\end{aligned}
$$

When the seasonal mean and post-optimality values in Figure 4 are superposed with the mixed-strategy game model tariff estimate value, we have Figure 5:

\subsection{Summer Time-Block Sensitivity Analysis Results}

The following time-block optimal range of values in Table 8 coinciding with the same optimal time-blocks in the game model are obtained from the Dual problem (using the LPSolve software).

Using Equation (6), we obtain the corresponding post-optimality energy ranges by using Equation (13).

$$
T_{i}=\frac{t_{i}}{T_{s}}
$$

where $T_{i}$ is the Time-Block optimal mixed-strategy and $T_{s}=D_{s}=0.028355393$.

Thus the ranges of energy values are as follows:

$$
\begin{aligned}
& 35.16175215 \leq t_{16} \leq 36.32470197 \\
& 33.60693822 \leq t_{20} \leq 35.77974053 \\
& 34.27879848 \leq t_{39} \leq 35.30868593 \\
& 35.20508294 \leq t_{41} \leq 35.35365461
\end{aligned}
$$

\section{Duality Results and Discussions on Utility Application}

\subsection{Summer and Winter Dual Optimal Solutions}

Solving a linear programming problem usually provides more information about an optimal solution than merely the values of the decision variables. Associated with an optimal solution are shadow prices (also referred to as dual variables or marginal values) for the constraints. The shadow price on a particular constraint represents the change in the value of the objective function per unit increase in the right hand-side value of that constraint. Thus duality in linear programming is essentially a unifying theory that develops the relationships between a 


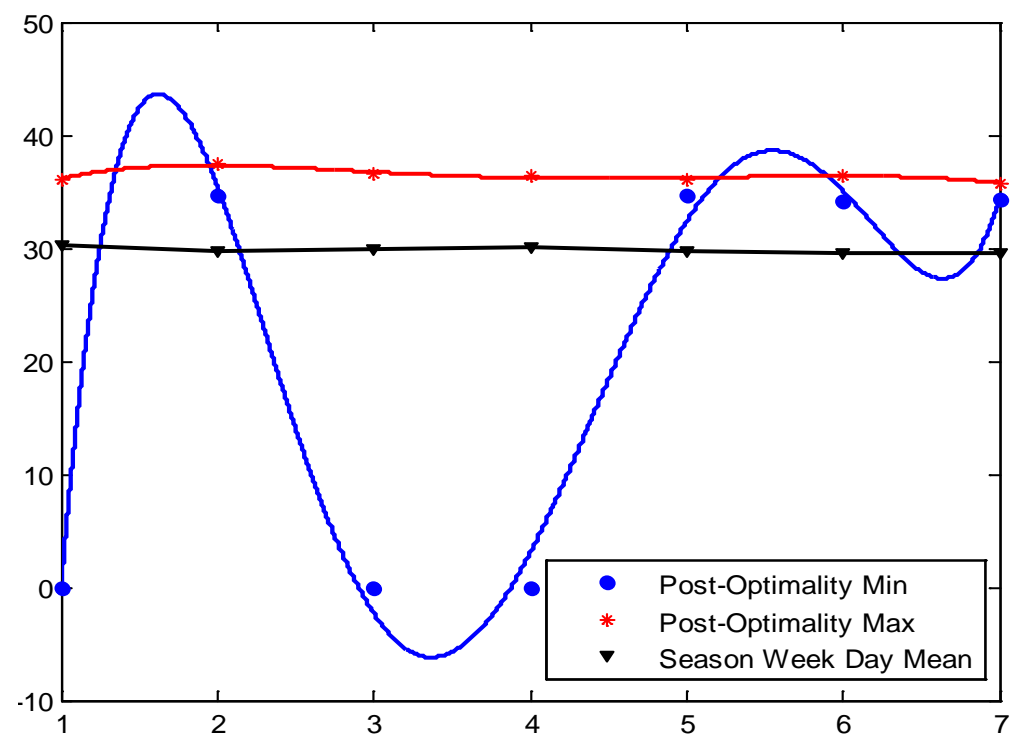

Figure 4. Relationships between the seasonal mean and post-optimality energy values.

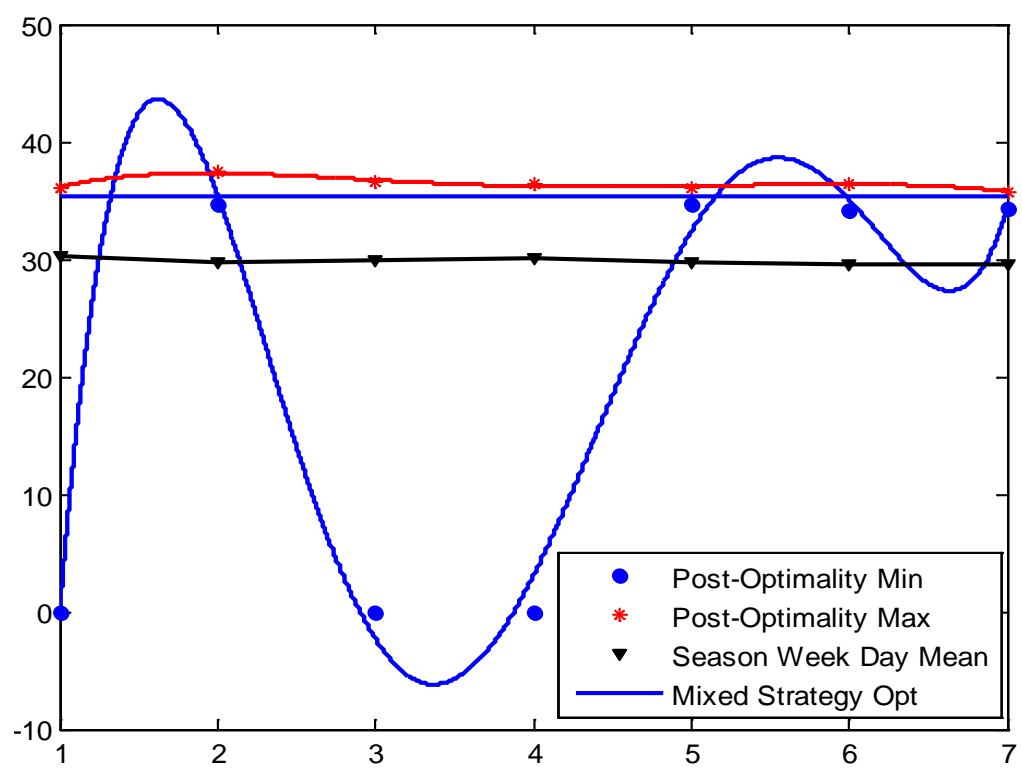

Figure 5. Relationships between the seasonal mean, post-optimality energy values and the tariff estimator.

Table 8. Results of summer dual problem sensitivity analysis.

\begin{tabular}{ccc} 
& From & Till \\
\hline Objective & 0.025019933136 & 0.02501993313 \\
\hline Variables & & \\
\hline$t_{16}=07 \mathrm{H} 30$ & 0.997025303 & 1.030001202 \\
$t_{20}=09 \mathrm{H} 30$ & 0.952937943 & 1.014548607 \\
$t_{39}=19 \mathrm{H} 00$ & 0.971988805 & 1.001191668 \\
$t_{41}=20 \mathrm{H} 00$ & 0.998253965 & 1.002466773 \\
\hline
\end{tabular}


given (primal) linear programming problem and another related (dual) linear programming problem stated in terms of variables with this shadow-price interpretation

The related dual problems to the week-day models for the two seasons have the time-blocks as the dual variables. In Figure 6 and Figure 7 we have the dual problem model solution yielding the time-block optimal energy values plotted against the seasonal season means, the seasons' Tariff Estimator game values and the four time-optimal energy consumption values for the winter and summer seasons, respectively.

\subsection{Discussions on Utility Applications}

From the foregoing model results, the following conclusions are drawn:

1) The direct LPP models and their associated post-optimality analyses for the two seasons validate our earlier optimal mixed strategy game values, both for the week-day and time-block optimal values.

2) The post-optimality analysis for each of the seasons provides additional information on the non-optimal week-day mixed strategies, specifically, the analysis shows the significant maximum energy values which are

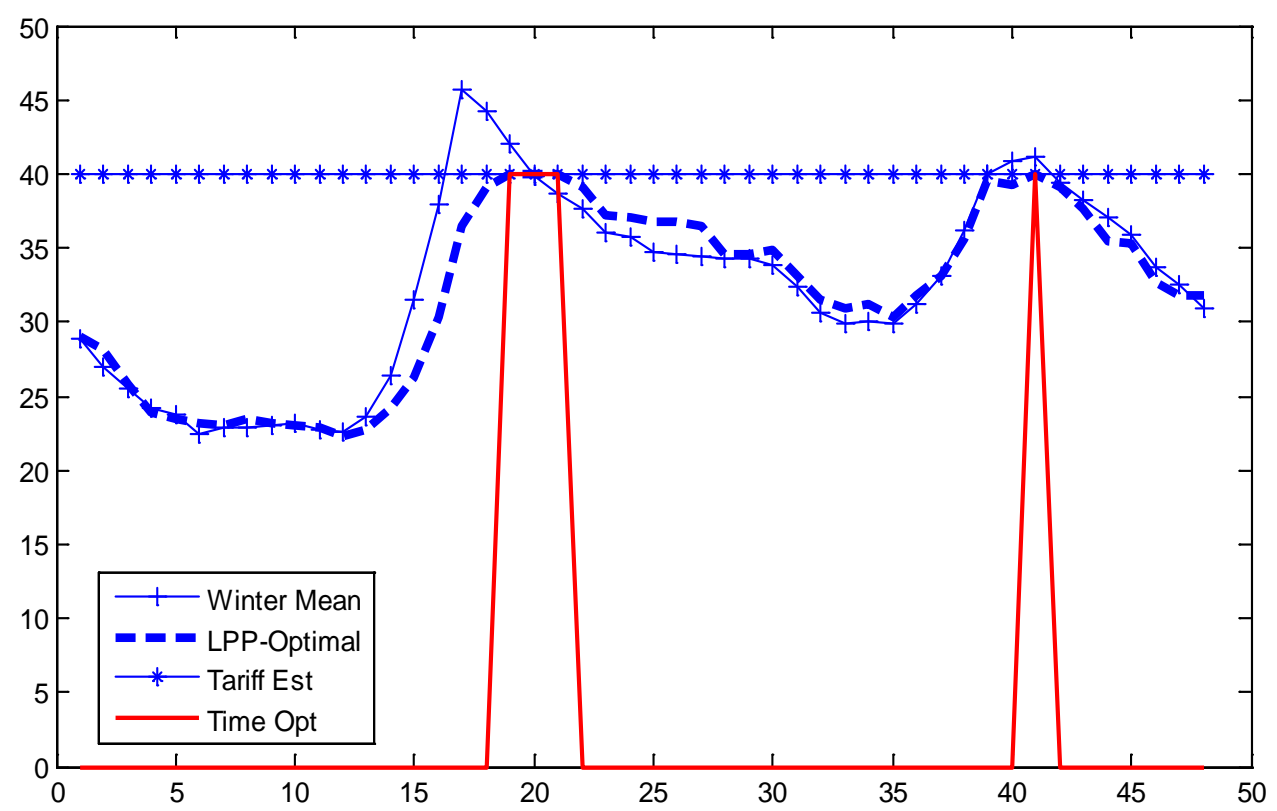

Figure 6. Winter comparison plots and dual optimal simulation.

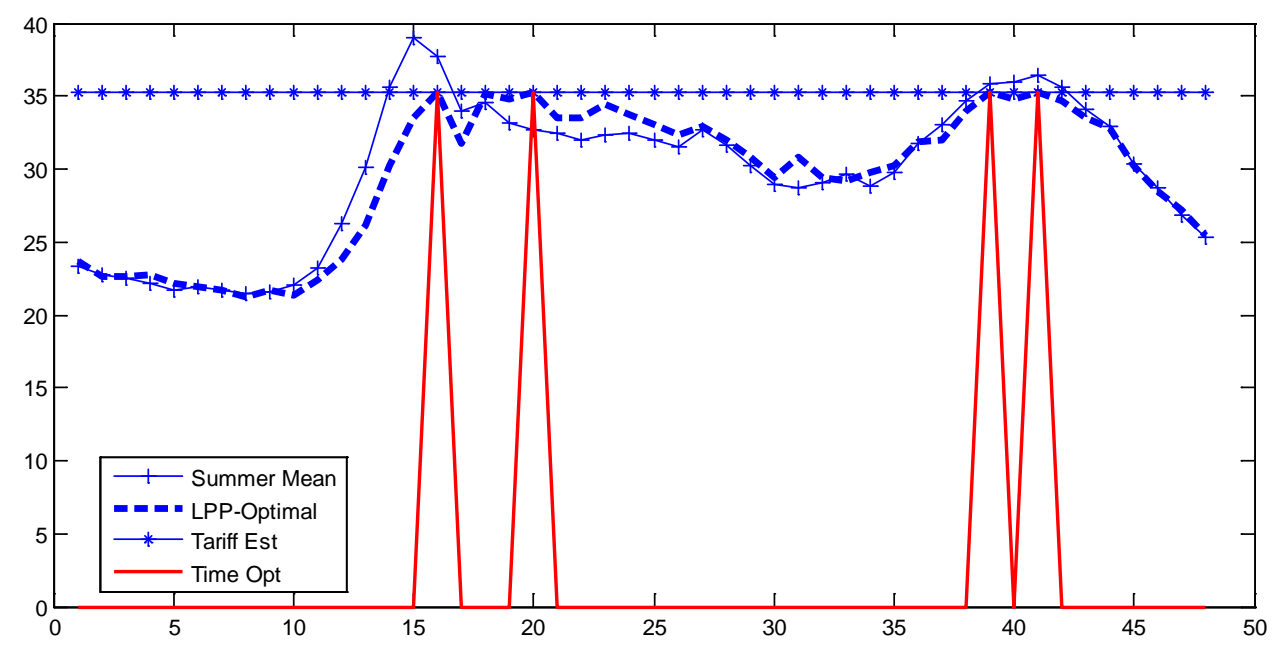

Figure 7. Summer comparison plots and dual optimal simulation. 
not explicit in the game model results as summarised in Table 9:

3) In each of the seasons, the tariff energy estimator obtained from the mixed-strategy model lies within the post-optimality ranges. This is clearly seen in the plots in Figure 2 and Figure 5. Thus this confirms the uniqueness of the estimator as a very useful energy value for utility application, especially for determining a single uniform tariff estimate.

4) While the Time-Block post-optimality results may not be of significant application from utility point of view, however, they equally validate our mixed-strategy game model values, and also specifically show the range of energy values within the optimal time-blocks that were not explicit from the game model.

5) It is noteworthy to see that the time-optimal solution of the dual problem model reveals small deviations from each of the season's means, globally.

We had earlier [1] categorized the consumers into morning (workplace) and (evening) residential consumers. Thus the comparison plots in Figure 8 show two fairly convergence points for the two seasons that appear useful for some estimates for the two periods of the day for the whole year.

\section{Conclusion}

Utilities, i.e. agencies in charge of distribution of electricity commonly face a serious challenge of determining a tariff policy as noted in [5]. This is even more challenging for consumers where provision of electricity meters (or metering) is not easily feasible, such as the informal settlements. Having determined a tariff estimate in our earlier game model as in [1], useful for utilities in determining a uniform tariff policy, knowing the range of energy demand (or consumption) values within which uniform tariff remains unchanged, becomes essential for

Table 9. Summary of seasonal sensitivity analyses.

\begin{tabular}{ccc}
\hline \multirow{2}{*}{ Week-Day } & Winter Post-Optimality Week-Day Energy Range & Summer Post-Optimality Week-Day Energy Range \\
\cline { 2 - 3 } & Tariff Estimator $=39.9681$ & Tariff Estimator $=35.2666$ \\
\hline Monday & $0 \leq X_{1} \leq 41.3948284$ & $0 \leq X_{1} \leq 36.12104245$ \\
Tuesday & $0 \leq X_{2} \leq 41.72583365$ & $34.61555364 \leq X_{2} \leq 37.40478333$ \\
Wednesday & $0 \leq X_{3} \leq 40.29555199$ & $0 \leq X_{3} \leq 36.54649026$ \\
Thursday & $39.75519492 \leq X_{4} \leq 40.56906598$ & $0 \leq X_{4} \leq 36.4700509$ \\
Friday & $39.61080734 \leq X_{5} \leq 40.28072835$ & $34.64216239 \leq X_{5} \leq 36.02510706$ \\
Saturday & $39.42965009 \leq X_{6} \leq 40.19669252$ & $34.20039587 \leq X_{6} \leq 36.47142456$ \\
Sunday & $39.50194189 \leq X_{7} \leq 40.86035541$ & $34.37565093 \leq X_{7} \leq 35.78001817$ \\
\hline
\end{tabular}

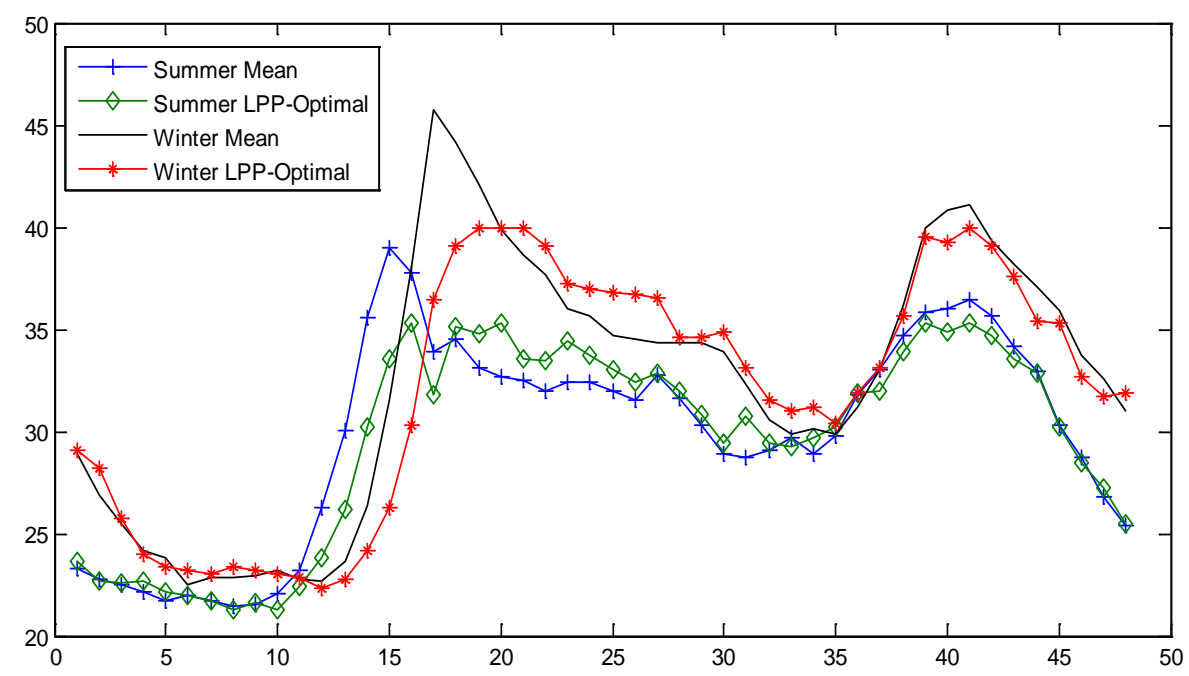

Figure 8. Seasonal means and the dual LPP time optimal values. 
utility break-even analysis. This requires a post-optimality (or sensitivity) analysis as presented in this paper and reflected in the abstract.

\section{Acknowledgements}

The authors wish to acknowledge the partial support of this research work by the Polytechnic of Namibia's Institutional Research and Publication Committee (IRPC).

\section{References}

[1] Gope, G., Reju, S.A. and Kanyimba, K. (2012) Residential Time of Use Energy Modelling and Tariff Evaluation. Canadian Journal on Electrical and Electronics Engineering, 3, 115-120.

[2] Gope, G., Reju, S.A. and Kamanja, T. (2013) A Review of Energy Modelling Methods and the Application of Wireless Sensor Networks (WSNs). Proceedings of Pan African International Conference on Information Science, Computing and Telecommunications (PACT 2013), 15-18 July 2013, Lusaka, 50-55.

[3] Arsham, H. (2011) Construction of General Sensitivity Regions. http://home.ubalt.edu/ntsbarsh/Business-stat/opre/PartVII.htm

[4] Bradley, S.P., Hax, A.C. and Magnanti, T.L. (1977) Applied Mathematical Programming. Addison-Wesley, Boston. http://web.mit.edu/15.053/www/AMP-Chapter-03.pdf

[5] Li, Y. and Cui, J. (2008) A Method of Designing Energy Tax Rate Based on Game Theory. The 7th International Symposium on Operations Research and Its Applications (ISORA'08), Lijiang, 31 October-3 November 2008. 
Scientific Research Publishing (SCIRP) is one of the largest Open Access journal publishers. It is currently publishing more than 200 open access, online, peer-reviewed journals covering a wide range of academic disciplines. SCIRP serves the worldwide academic communities and contributes to the progress and application of science with its publication.

Other selected journals from SCIRP are listed as below. Submit your manuscript to us via either submit@scirp.org or Online Submission Portal.
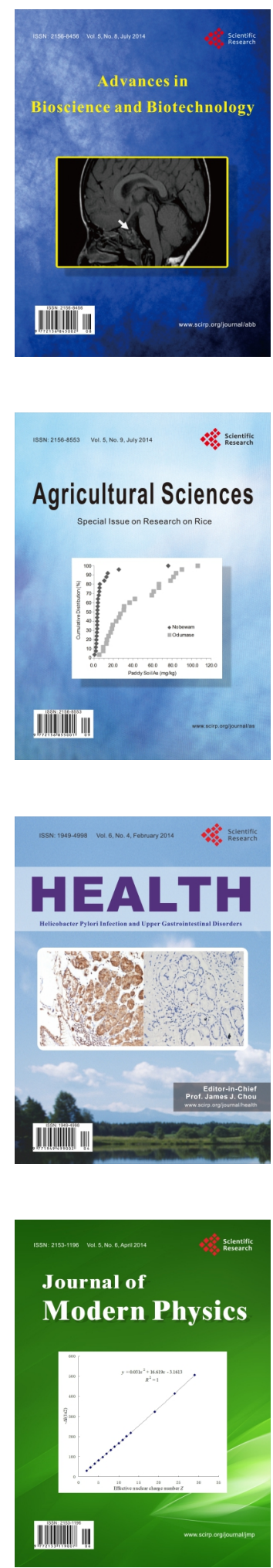
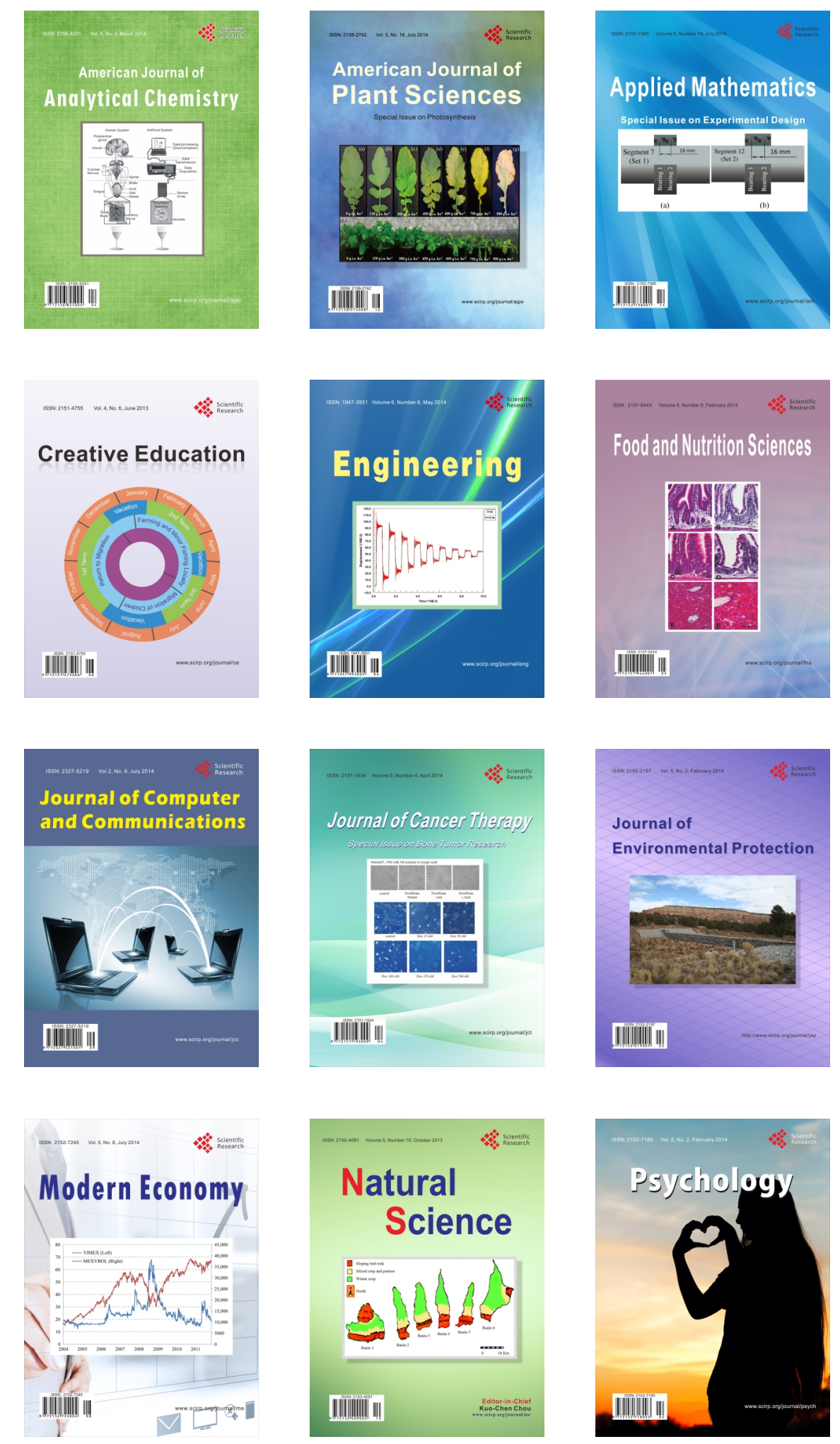